\title{
Anti-photocorrosive photoanode with RGO/PdS as hole extraction layer
}

\author{
Guo-Qiang Liu ${ }^{1 \dagger}$, Yi $\mathrm{Li}^{1 \dagger}$, Yuan Yang ${ }^{1}$, Feng-Jia Fan ${ }^{2}$, Guang-Hao Ding ${ }^{1}$, Liang Wu ${ }^{1}$, Jun Hu ${ }^{3}$, \\ Jin-Lan Peng ${ }^{4}$, Qian $\mathrm{Xu}^{3}$, Jun-Fa $\mathrm{Zhu}^{3}$ and Shu-Hong $\mathrm{Yu}^{{ }^{*}}$
}

\begin{abstract}
Photoelectrochemical (PEC) hydrogen production is of great interest as an ideal avenue towards clean and renewable energy. However, the instability and low energy conversion efficiency of photoanodes hinder their practical applications. Here we address these issues by introducing a hole extraction layer (HEL) which could rapidly transfer and consume photogenerated holes. The HEL is constructed by reduced graphene oxide (RGO) and other cocatalysts that enable rapid transfer and subsequent consumption of holes, respectively. Specifically, we showcase a high-stability photoanode composed of CdSeTe nanowires (CST NWs) and RGO/ PdS nanoparticles (PdS NPs) based HEL. The photoanode achieves excellent photocorrosion resistance, which allows stable hydrogen evolution for $>2 \mathrm{~h}$ at $0.5 \mathrm{~V}_{\mathrm{RHE}}$.
\end{abstract}

Keywords: CdSeTe, photoelectrochemistry, hole extraction layer, photocorrosion, photoanode

\section{INTRODUCTION}

Photoelectrochemical (PEC) hydrogen production provides a highly promising and eco-friendly way of solving the energy crisis [1-13]. Among multitudinous semiconductors, II-VI semiconductors $\mathrm{CdX}(\mathrm{X}=\mathrm{Se}, \mathrm{Te})$ with energy band gaps of 1.4-1.7 eV have strong absorptions for visible and near-infrared light [14-16]. The suitable band gap and high absorption coefficient $\left(\alpha, 10^{5} \mathrm{~cm}^{-1}\right)$ endow these materials with the potential to act as efficient photoelectrodes [16-19].

Unfortunately, the cadmium chalcogenides, such as CdTe and CdSe, often suffer from a detrimental photocorrosion due to the photogenerated holes, resulting in severe performance degradation during operation [2022]. The photocorrosion in chalcogenide semiconductors origins from poor hole transfer and the subsequent sluggish redox reactions at photoelectrode-electrolyte interface.

To date, there are two approaches to prevent the photocorrosion of cadmium chalcogenides. One is to introduce physical barriers, such as an inert passivation layer (for instance, $\mathrm{SiO}_{2}$ and $\mathrm{TiO}_{2}$ ), that can isolate semiconductors from the electrolyte [23,24]. However, the concomitantly retarded interfacial charge transfer and blocked surface reaction sites discount their solar conversion efficiency. The other is to accelerate hole transfer and subsequent redox reactions with electrolytes to a level kinetically competitive to the photocorrosion process. The most common strategy in this respect is to introduce hetero/homojunctions for hole transfer [23,25,26], cocatalysts for hole-involved reactions [27-29], and combinations thereof, giving rise to some encouraging results. Whereas, efforts in improving the solar conversion efficiency and anti-photocorrosion capability of CdSe/CdTebased photoanodes remains unsatisfying, due to the absence of suitable hole extraction layers (HELs).

\footnotetext{
${ }^{1}$ Division of Nanomaterials \& Chemistry, Hefei National Laboratory for Physical Sciences at the Microscale, Institute of Energy, Hefei Comprehensive National Science Center, CAS Center for Excellence in Nanoscience, Department of Chemistry, Institute of Biomimetic Materials \& Chemistry, University of Science and Technology of China, Hefei 230026, China

${ }^{2}$ CAS Key Laboratory of Microscale Magnetic Resonance and Department of Modern Physics, University of Science and Technology of China, Hefei 230026, China

${ }^{3}$ National Synchrotron Radiation Laboratory, University of Science and Technology of China, Hefei 230029, China

${ }^{4}$ Center for Micro- and Nanoscale Research and Fabrication, University of Science and Technology of China, Hefei 230026, China

† These authors contributed equally to this work.

* Corresponding author (email: shyu@ustc.edu.cn)
} 
We, therefore, sought to develop an HEL constructed by hole conductor and oxidation cocatalysts, as an approach to enhance the stability of photoelectrodes. Reduced graphene oxide (RGO) nanosheets, where aromatic $\mathrm{sp}^{2}$ domains with few nanometers are surrounded by $\mathrm{sp}^{3}$ hybridized carbon atoms, can serve as a compelling hole transport medium to improve the charge carrier transfer kinetics [30] thanks to its unique electronic properties, large surface area, and excellent optical transmittance $[31,32]$. RGO can effectively improve the carrier transport efficiency of photoelectrodes and reduce the carrier recombination rate. In addition, the synergistic effect of RGO and cocatalyst can accelerate the transfer and consumption of photogenerated carriers at the same time, further ensuring the stability of photoelectrodes. Through combining the hole-transporting ability of RGO and the hole-depleting ability of oxidation cocatalysts, the RGO/ oxidation cocatalyst based HEL is expected to enhance the photostability and solar conversion efficiency simultaneously.

Following such HEL strategy, here we designed a highly stable photoanode which consists of CdSeTe nanowires (CST NWs) and RGO/PdS-based HEL. The RGO and PdS nanoparticles (NPs) were used to rapidly transport and accelerate hole consumption, thereby suppressing the photocorrosion of photoanode, as illustrated in Fig. 1. We studied the charge transfer kinetics of these photoanodes, which were consistent with the PEC stability performance. As a result, the photoanode achieved a photocurrent density of $1.6 \mathrm{~mA} \mathrm{~cm}{ }^{-2}$ at $0.5 \mathrm{~V}_{\mathrm{RHE}}$ under 1-sun light, and only decayed by $7 \%$ after $2 \mathrm{~h}$ operation-the figure of merits significantly superior to bare CST NWs, of which the photocurrent density was only $0.2 \mathrm{~mA} \mathrm{~cm}^{-2}$ and further decayed by $50 \%$ after $2 \mathrm{~h}$ operation.

\section{EXPERIMENTAL SECTION}

\section{Materials}

$\mathrm{Na}_{2} \mathrm{TeO}_{3}$ (98\%) was purchased from Maya Reagent (China). Polyvinylpyrrolidone (PVP, K30), Se powder (99\%), aqueous ammonium solution (25-28 w\%), hydrazine hydrate $(85 \mathrm{w} \%)$, acetone, $\mathrm{Cd}\left(\mathrm{NO}_{3}\right)_{2} \cdot 4 \mathrm{H}_{2} \mathrm{O}$, thiourea, $\mathrm{HPdCl}_{4}\left(25 \mathrm{mmol} \mathrm{L}^{-1}\right), \mathrm{N}, \mathrm{N}$-dimethylformamide (DMF), trichloromethane $\left(\mathrm{CHCl}_{3}\right)$, sodium sulfide $\left(\mathrm{Na}_{2} \mathrm{~S}\right)$ and sodium sulfite $\left(\mathrm{Na}_{2} \mathrm{SO}_{3}\right)$ were purchased from Shanghai Reagent Company (China). All chemicals were used as received without further purification.

\section{Instruments}

The morphology of nanostructures was investigated by

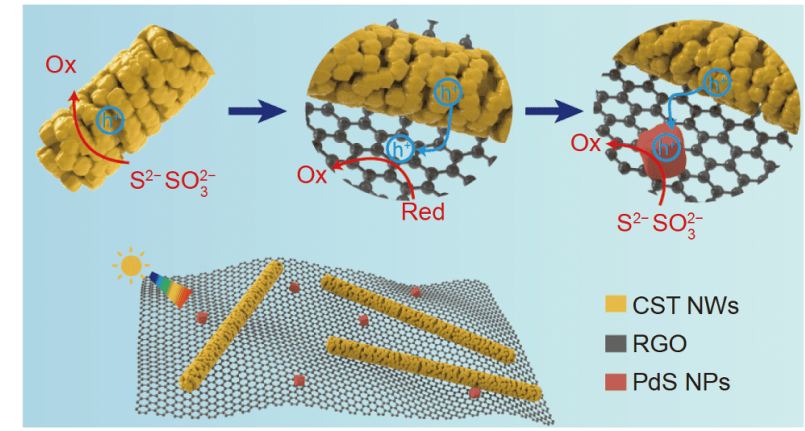

Figure 1 Schematic illustration of the HEL-based photoanode. The anti-photocorrosion photoanodes are constructed by CST NWs and PdS NPs which are uniformly dispersed on RGO. There are three pathways for hole consumption: the first one is directly consumed by hole scavengers at the surface of CST NWs; the second one is transported by RGO and then consumed by its functional group; and the last one is consumed by PdS NPs where holes are transferred from CST NWs passing through RGO.

transmission electron microscopy (TEM) using a Hitachi $\mathrm{H}-7700$ microscope at $100 \mathrm{kV}$. Scanning electron microscopy (SEM) images were obtained with a field emission scanning electron microanalyzer (Zeiss Supra 40) at an acceleration voltage of $5 \mathrm{kV}$. X-ray diffraction (XRD) patterns were measured on a Japan Rigaku DMax- $\gamma$ A rotation anode $\mathrm{X}$-ray diffractometer equipped with graphite monochromatized $\mathrm{Cu}-\mathrm{K}$ radiation $(\lambda=1.54178 \AA$ ). High Resolution TEM (HRTEM), high-angle annular dark-field scanning TEM (HAADF-STEM) image and energy dispersive spectra (EDS) elemental mapping were obtained by JEOL 2100F, FEI Tecnai G2 F20 S-Twin microscope at $200 \mathrm{kV}$. X-ray photoelectron spectroscopy (XPS) was recorded using an ESCALAB-MKII X-ray photoelectron spectrometer equipped with $\mathrm{Mg} \mathrm{Ka}$ radiation as an exciting source. Raman spectra were detected by a Renishaw System 2000 spectrometer using the $514.5 \mathrm{~nm}$ line of $\mathrm{Ar}^{+}$for excitation. UV-vis-NIR absorption spectra were obtained by a Shimadzu UV3600 spectrometer at room temperature. Atomic force microscopy (AFM) was performed by Bruker dimension icon. The infrared spectra (IR) were carried out on a NICOLET Fourier transform infrared spectrometer using pressed $\mathrm{KBr}$ tablets. Thermal gravimetric analysis (TGA) of the samples was obtained on a Shimadzu TA-50 thermal analyzer (Shimadzu) at a heating rate of $10^{\circ} \mathrm{C} \mathrm{min}^{-1}$ from room temperature to $1200^{\circ} \mathrm{C}$. In addition, ultraviolet photoelectron spectroscopy (UPS) spectra were performed at the Catalysis and Surface Science Endstation (BL11U beamline) in the National Synchrotron Radiation Laboratory (NSRL) in Hefei, China. A sample bias of 
$-5 \mathrm{~V}$ was applied to observe the secondary electron cutoff and the photon energy was $40.0 \mathrm{eV}$. The work function $(\Phi)$ was determined by the difference between the photon energy and the binding energy of the secondary cutoff edge. In actual test, the Au layer was assigned to $0 \mathrm{eV}$ and acted as a reference for $E_{\mathrm{f}}$.

\section{Synthesis of CdSeTe ternary alloy NWs}

According to our previous work [33], Te $\mathrm{Se}_{y} @ \mathrm{Se}$ NWs can be obtained by using Te NWs as templates. $0.9 \mathrm{mmol}$ of $\mathrm{Cd}\left(\mathrm{NO}_{3}\right)_{2} \cdot 4 \mathrm{H}_{2} \mathrm{O}, 40 \mathrm{~mL}$ of deionized water (DIW) and $0.9 \mathrm{mmol}$ of $\mathrm{Te}_{x} \mathrm{Se}_{y} @ \mathrm{Se} \mathrm{NWs}$ solution ([Te]:[Se]=1:6) were mixed in a $100-\mathrm{mL}$ Teflon-lined stainless steel autoclave under magnetic stirring. Then the autoclave was sealed and heated at $160^{\circ} \mathrm{C}$ for $12 \mathrm{~h}$. After the reaction, the final products could be collected by centrifugation (10,000 rpm, $3 \mathrm{~min})$ and washed thrice with ethanol.

\section{Synthesis of CdSeTe/RGO}

In the synthesis of $\mathrm{CdSeTe} / \mathrm{RGO}$, a varying amount of GO solution $\left(0.35 \mathrm{mg} \mathrm{mL}^{-1}\right)$ was dispersed with $80 \mathrm{~mL}$ of DIW to form a homogenous solution by ultrasound. Then, $0.9 \mathrm{mmol}$ CST NWs solution was added to the GO solution. The solution mixture was stirred magnetically for $30 \mathrm{~min}$ and transferred into an autoclave and heated to $160^{\circ} \mathrm{C}$ for $12 \mathrm{~h}$. After the reaction, the final products should be collected by centrifugation (6000 rpm, $3 \mathrm{~min}$ ) and washed three times with ethanol.

\section{Synthesis of CdSeTe/RGO/PdS}

$\mathrm{HPdCl}_{4}\left(3 \mathrm{~mL}, 25 \mathrm{mmol} \mathrm{L}^{-1}\right), 1 \mathrm{~g}$ of thiourea, and $80 \mathrm{~mL}$ DIW were mixed in a $100-\mathrm{mL}$ Teflon-lined stainless steel autoclave under magnetic stirring. Then the autoclave was sealed and maintained at $160^{\circ} \mathrm{C}$ for $12 \mathrm{~h}$. After the reaction, the final products should be collected by centrifugation $(10,000 \mathrm{rpm}, 3 \mathrm{~min})$ and washed thrice with ethanol. A varying amount of PdS NPs solution was collected by centrifugation $(10,000 \mathrm{rpm}, 3 \mathrm{~min})$ to extract the PdS NPs. $80 \mathrm{~mL}$ CST/RGO solution and PdS NPs above were mixed in a $100-\mathrm{mL}$ Teflon-lined stainless steel autoclave under magnetic stirring. Then the autoclave was sealed and maintained at $160^{\circ} \mathrm{C}$ for $12 \mathrm{~h}$. After the reaction, the final products should be collected by centrifugation $(10,000 \mathrm{rpm}, 3 \mathrm{~min})$ and washed thrice with ethanol.

\section{Synthesis of CdSeTe/PdS}

CST NWs solution $(80 \mathrm{~mL})$ was collected by centrifugation $(10,000 \mathrm{rpm}, 3 \mathrm{~min})$ to extract the CST NWs. The CST NWs were redispersed in $80 \mathrm{~mL}$ DIW to form a homogenous solution. After that, a varying amount of $\mathrm{HPdCl}_{4}\left(25 \mathrm{mmol} \mathrm{L}^{-1}\right), 0.1 \mathrm{~g}$ of thiourea and CST NWs solution were mixed in a $100-\mathrm{mL}$ Teflon-lined stainless steel autoclave under magnetic stirring. Then the autoclave was sealed and maintained at $160^{\circ} \mathrm{C}$ for $3 \mathrm{~h}$. After the reaction, the final products should be collected by centrifugation $(10,000 \mathrm{rpm}, 3 \mathrm{~min})$ and washed thrice with ethanol.

\section{Femtosecond transient absorption (TA) measurement}

We used CST, CST/RGO, CST/PdS, and CST/RGO/PdS films for transient absorption measurement. For the TA measurement (Time-Tech Spectra, TA100), the fundamental output from Yb:KGW laser $(1030 \mathrm{~nm}, 100 \mathrm{kHz}$, 220 fs Gaussian fit, Light Conversion Ltd.) was separated to two light beams. One beam was introduced to the optical parametric amplifier (ORPHEUS-N, Light Conversion Ltd.) to produce $450 \mathrm{~nm}$ pump beam, and the other was focused onto a YAG plate to produce white light continuum probe beam. A linear array detector was used to collect the transmitted probe light from the sample.

\section{PEC measurement}

PEC performances of the photoanodes were performed on a CHI760D electrochemistry workstation in a typical three-electrode configuration with a $\mathrm{Pt}$ foil and $\mathrm{Ag} / \mathrm{AgCl}$ as the counter electrode and the reference electrode, respectively. The simulated solar illumination was offered by passing light from a $300 \mathrm{~W}$ xenon lamp equipped with a UV light filter $(\lambda>420 \mathrm{~nm})$. The intensity of the incident light was calibrated to $100 \mathrm{~mW} \mathrm{~cm}^{-2}$ by a power meter. Photocurrent measurements were measured in an electrolyte solution including $0.08 \mathrm{~mol} \mathrm{~L}^{-1} \quad \mathrm{Na}_{2} \mathrm{~S}$ and $0.50 \mathrm{~mol} \mathrm{~L}^{-1} \mathrm{Na}_{2} \mathrm{SO}_{3}$ as hole scavenger ( $\mathrm{pH}$ 12.86). The electrolyte was thoroughly deaerated by purging with nitrogen before measurements. The photocurrents were measured at a range of -1.2 to $0.2 \mathrm{~V}$ vs. $\mathrm{Ag} / \mathrm{AgCl}$ with a scan rate of $10 \mathrm{mV} \mathrm{s}^{-1}$. The Mott-Schottky plot was collected with a CHI760D electrochemistry workstation through an impedance-capacitance scan technique with an amplitude of $10 \mathrm{mV}$. The electrolyte and the photoanodes were the same as that in PEC current measurements. Incident photon-to-electron conversion efficiency (IPCE) was also measured with the same workstation and in the same electrolyte with a threeelectrode configuration at $0.5 \mathrm{~V}_{\mathrm{RHE}}$, from 380 to $900 \mathrm{~nm}$. All of the electrode potentials were recorded with $\mathrm{Ag} /$ $\mathrm{AgCl}$ electrode and transformed to a reversible hydrogen electrode (RHE). 


\section{RESULTS AND DISCUSSION}

$\mathrm{CdSe}, \mathrm{CdTe}$, and their alloy compounds are very suitable for various optoelectronic devices, such as PEC cells and solar cells, due to the suitable bandgaps and high optical absorption coefficients [15,19]. The ternary semiconductor $\mathrm{CdSe}_{x} \mathrm{Te}_{1-x}$ possesses adjustable band gaps along with the change of $x$, which enables readily engineered band structures for the PEC hydrogen production [15]. However, the photocorrosion seriously hinders the practical application of these materials.

To address this issue, PdS NPs as oxidation cocatalysts were dispersed on RGO to form an HEL. In order to accurately explore the capability of RGO/PdS-based HEL in enhancing the stability of CST-based photoanodes, we prepared various types of composites using CST NWs, RGO, and PdS NPs as building blocks [33,34].

As shown by low-magnification TEM and SEM characterization, the CST NWs were constructed by small NPs of $5-10 \mathrm{~nm}$, which were synthesized by chemical transformation from $\mathrm{Te}_{x} \mathrm{Se}_{y} @ \mathrm{Se}$ core-shell NWs (Figs S1, S2a, and S3a). Besides, we also prepared the CST/RGO composites by loading CST NWs onto GO nanosheets through ultrasonic dispersion and hydrothermal treatment (Figs S2b and S3b). The CST/RGO/PdS was formed by further decorating PdS NPs onto CST/RGO (Fig. 2a, Figs S3c and S4). As shown in the TEM and SEM images, the CST NWs as well as PdS NPs were uniformly dispersed on RGO in the CST/RGO/PdS composites. CST/ $\mathrm{PdS}$ composites were formed when the CST NWs were only decorated with PdS NPs (Figs S2c and S3d).

We further characterized the ultrathin features of the nanosheets by atomic force microscopy (AFM), which showed a thickness of only 1-2 nm (Fig. 2b). Such ultrathin RGO can increase its optical transmittance, avoiding blocked optical absorption of CST NWs. The crystal structures of these samples were verified by powder XRD. All diffraction peaks in the XRD pattern of CST NWs can be indexed to the wurtzite CdSe (JCPDS card No. 08-0459, space group of P63mc) and zinc blende-phase CdSe (JCPDS card No. 19-0191, space group of $F-43 m$ ) with an overall shift to lower $2 \theta$ values due to the incorporation of tellurium (Fig. S5a). The peaks between $2 \theta$ of $30^{\circ}$ and $32^{\circ}$ are indexed to the tetragonal-phase PdS (JCPDS card No. 25-1234, space group of $P 42 / m$ ), which is in line with the XRD pattern of PdS NPs. Correspondingly, the HRTEM image (Fig. 2c) shows lattice spacing of $0.36 \mathrm{~nm}$, which can be indexed to the (100) planes of the wurtzite CdSe. The lattice spacings of $0.22 \mathrm{~nm}$ of PdS NPs can be indexed to the (212) planes (Fig. 2d). Beside, according to the HRTEM image of CST/
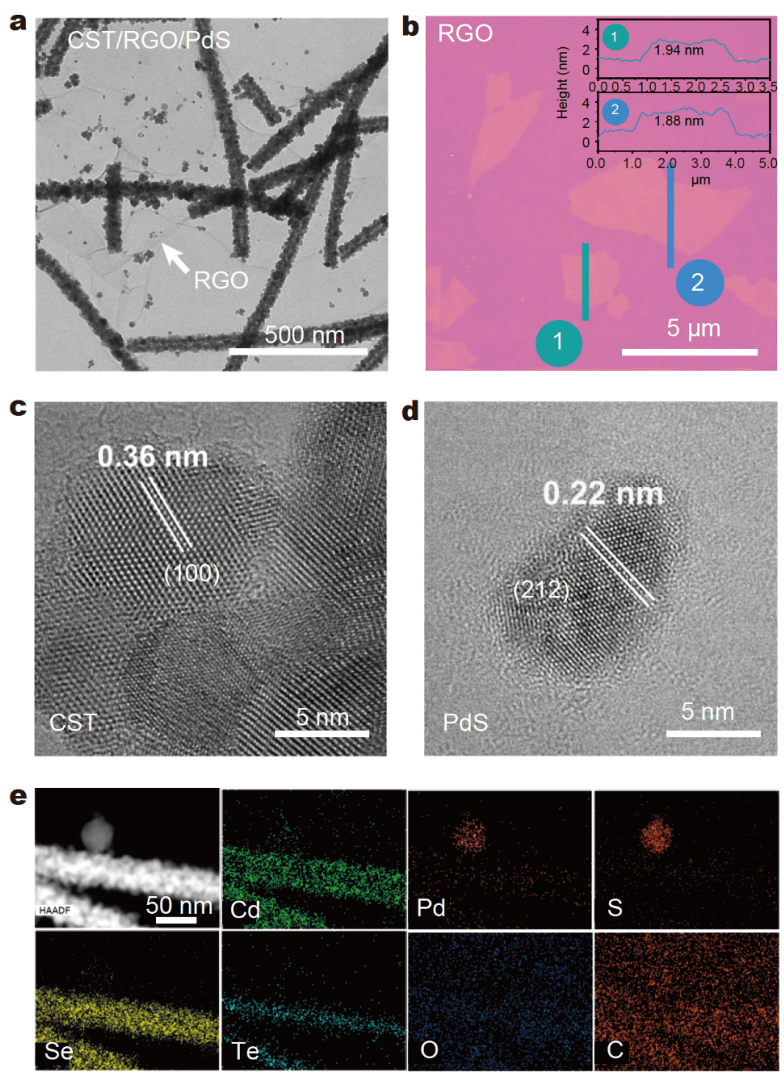

Figure 2 (a) TEM image of CST/RGO/PdS. (b) AFM image of RGO. Inset is the thickness profile. HRTEM images of CST NWs (c) and PdS NPs (d), respectively. (e) EDS elemental mapping images of CST/RGO/ PdS.

PdS, the PdS NPs adhere to the CST NWs (Fig. S6).

The EDS elemental mapping images of the CST/RGO/ PdS (Fig. 2e) reveal that the $\mathrm{Cd}$ and Se elements are homogeneously distributed in the NWs, while Te is mainly distributed in the core of NWs due to the coreshell structure of the $\mathrm{Te}_{x} \mathrm{Se}_{y} @ \mathrm{Se}$ nanowire template [33]. Moreover, the Pd and S are homogeneously distributed in the PdS NPs. The subsequent annealing process is necessary to improve the crystallinity of NWs as well as remove the long-chain PVP ligands from the NW surface. Further characterizations indicate that annealing has no influences on the morphologies and crystal structures of the photoanode materials except that Te distributes uniformly in NWs after annealing (Figs S7-S14). We determined the surface compositions of these samples by XPS (Figs S15-S18). The peaks of CST/RGO and CST/ $\mathrm{RGO} / \mathrm{PdS}$ at $283.0 \mathrm{eV}$ can be assigned to the carbonmetal coordinate bond, indicating strong electronic interactions in $\mathrm{RGO} / \mathrm{CST}$ as well as $\mathrm{RGO} / \mathrm{PdS}$, which is conducive to improving the ultrafast transport of pho- 
togenerated electrons in semiconductor and graphene.

In order to investigate the band alignment of these samples, we took detailed spectroscopic characterizations on CST NWs and PdS NPs, and characterized their optical band gaps $\left(E_{\mathrm{g}}\right)$ by UV-vis diffuse spectra, which were transformed into the Tauc plot (Fig. S19). The optical band gaps of CST and PdS are 1.60 and $1.70 \mathrm{eV}$, respectively. In contrast to the band gap of pure CdSe $(1.75 \mathrm{eV})$, the CST NWs show smaller optical band gap owing to the doping of Te. Using UPS, we precisely determined the band structures of CST NWs and PdS NPs. According to the equation $\Phi=h v-E_{\text {onset }}$, the work functions $(\Phi$, the relative energy gap between Fermi level and vacuum level) of CST and PdS are 4.0 and $4.3 \mathrm{eV}$, respectively (Fig. 3a). As shown in Fig. 3b, the relative electron binding energies between the valence band maximum and Fermi level $\left(E_{\mathrm{VBM}}-E_{\mathrm{f}}\right)$ of CST and PdS are -1.3 and $-1.5 \mathrm{eV}$, respectively. Given the relationship between $E_{\mathrm{g}}, E_{\mathrm{VBM}}-E_{\mathrm{f}}$, and $\Phi$, Fig. $3 c$ presents the energy band diagrams of CST and PdS. The $E_{\mathrm{VBM}}$ difference between CST NWs and PdS NPs drives photogenerated holes in CST NWs to flow into PdS NPs directly or through the hole-transporting RGO. In this way, the RGO/PdS NPs based HEL can significantly facilitate the charge separation and hole consumption. Under solar irradiation, photoexcited holes in CST NWs flow into RGO as well as PdS, and are further consumed by the redox reactions with electrolytes, therefore ensuring the stability of the photoanode.

In order to gain an in-depth understanding of the effects of RGO/PdS NPs based HEL, we further examined the charge carrier dynamics of CST, CST/RGO, CST/PdS, and CST/RGO/PdS with femtosecond-resolved TA spectroscopy. We used the pump-probe technique with a $450 \mathrm{~nm}$ pump and a white-light probe (550-800 $\mathrm{nm}$ ) to trace the photogenerated electron kinetics. Similar TA spectra were observed for all samples (Fig. S20). The results show that the lifetime of photoexcited electrons in the CST/RGO/PdS is much longer than other samples, a consequence that confirms the efficient charge separation and transfer process in ternary photoanode (Fig. 3d, Fig. S21, and Table S1). Combining the TA analyses and the energy band structures, we verified that the RGO/PdS NPs based HEL was capable of suppressing the photocorrosion of CST NWs and ensuring the conversion efficiency of the photoanodes.

To verify the advantages of RGO/PdS NPs based HEL in PEC hydrogen production, we examined their PEC
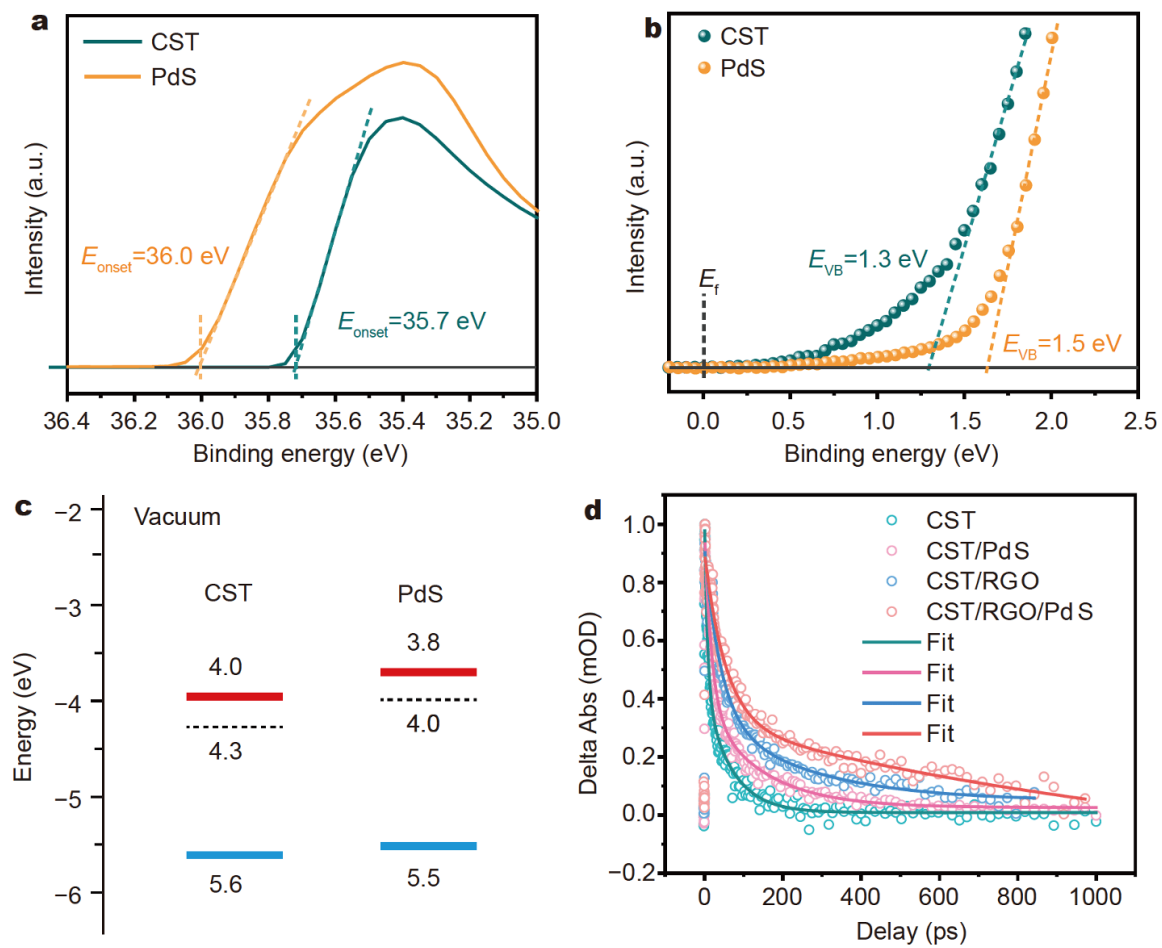

Figure 3 (a) Onset levels $\left(E_{\text {onset }}\right)$ of the UPS spectra for CST NWs and PdS NPs, respectively. (b) UPS spectra of CST NWs and PdS NPs, showing their relative energy gaps between valence band maximum and Fermi level. (c) Energy band diagrams of CST NWs and PdS NPs. (d) TA kinetics of CST, CST/PdS, CST/RGO, and CST/RGO/PdS excited by $450 \mathrm{~nm}$ pump. 
performances under illumination $(\lambda>420 \mathrm{~nm}$, $100 \mathrm{~mW} \mathrm{~cm}^{-2}$ ) with thickness-controlled photoanodes and using $\mathrm{Na}_{2} \mathrm{SO}_{3} / \mathrm{Na}_{2} \mathrm{~S}$ as hole scavengers [35,36] (Fig. S22). By adjusting the ratio of CST to RGO and CST to PdS, we optimized the performances of these photoanodes (Fig. S23). As shown in Fig. 4a, the anodic photocurrent density of CST/RGO/PdS increases at potentials above $-0.2 \mathrm{~V}_{\mathrm{RHE}}$, reaching $2.5 \mathrm{~mA} \mathrm{~cm}{ }^{-2}$ at $1.0 \mathrm{~V}_{\mathrm{RHE}}$. However, the photocurrent densities of CST, CST/RGO, and CST/PdS are $0.6,1.1$, and $1.5 \mathrm{~mA} \mathrm{~cm}^{-2}$ at $1.0 \mathrm{~V}_{\mathrm{RHE}}$, respectively. The improvement of PEC performances can be attributed to the fast hole transfer and consumption process assisted by RGO/PdS-based HEL.

We also collected the transient photocurrent responses of these photoanodes at a constant potential of $0.5 \mathrm{~V}_{\mathrm{RHE}}$ under 1-sun irradiation with a light chopper (Fig. S24a). The results show that CST/RGO/PdS photoanode exhibits a higher and more stable photocurrent density $\left(1.6 \mathrm{~mA} \mathrm{~cm}{ }^{-2}\right)$ than other photoanodes. Fig. $4 \mathrm{~b}$ shows that the CST/RGO/PdS photoanode possesses higher IPCE than CST, CST/PdS, and CST/RGO in the range of 380-900 nm. The IPCE of the CST/RGO/PdS photo- anode reaches $34 \%$ at $400 \mathrm{~nm}$ under an applied potential of $0.5 \mathrm{~V}_{\mathrm{RHE}}$. We estimated the carrier concentration in these photoanodes to be on the order of $10^{22} \mathrm{~cm}^{-3}$ using the Mott-Schottky plots [37,38] (Fig. 4c). The positive slopes of the Mott-Schottky curves confirm that all samples are of $\mathrm{N}$-type nature. The flat band potential $\left(V_{\mathrm{fb}}\right)$ of photoanodes composed of CST, CST/RGO, CST/ $\mathrm{RGO} / \mathrm{PdS}$, and CST/PdS were determined to be around $0.2 \mathrm{~V}_{\mathrm{RHE}}$. In addition, we used electrochemical impedance spectroscopy (EIS), which was carried out with the frequency of $10^{5}-0.1 \mathrm{~Hz}$ interval and an amplitude of $10 \mathrm{mV}$ at a bias potential of $0.5 \mathrm{~V}_{\mathrm{RHE}}$, to characterize the carrier transfer process. Fig. $4 \mathrm{~d}$ demonstrates that the semicircle of CST/RGO/PdS photoanode has a smaller diameter, implying that it has a lower charge transfer resistance $\left(R_{\mathrm{ct}}\right)$ than other samples $[39,40]$.

The anti-photocorrosion capability of CST, CST/RGO, CST/RGO/PdS, and CST/PdS photoanodes were studied by comparing the PEC photocurrent density decays of different samples before and after operation for a given time at $0.5 \mathrm{~V}_{\mathrm{RHE}}$ under 1-sun irradiation. As presented in Fig. 5, the CST/RGO/PdS photoanode shows a stable
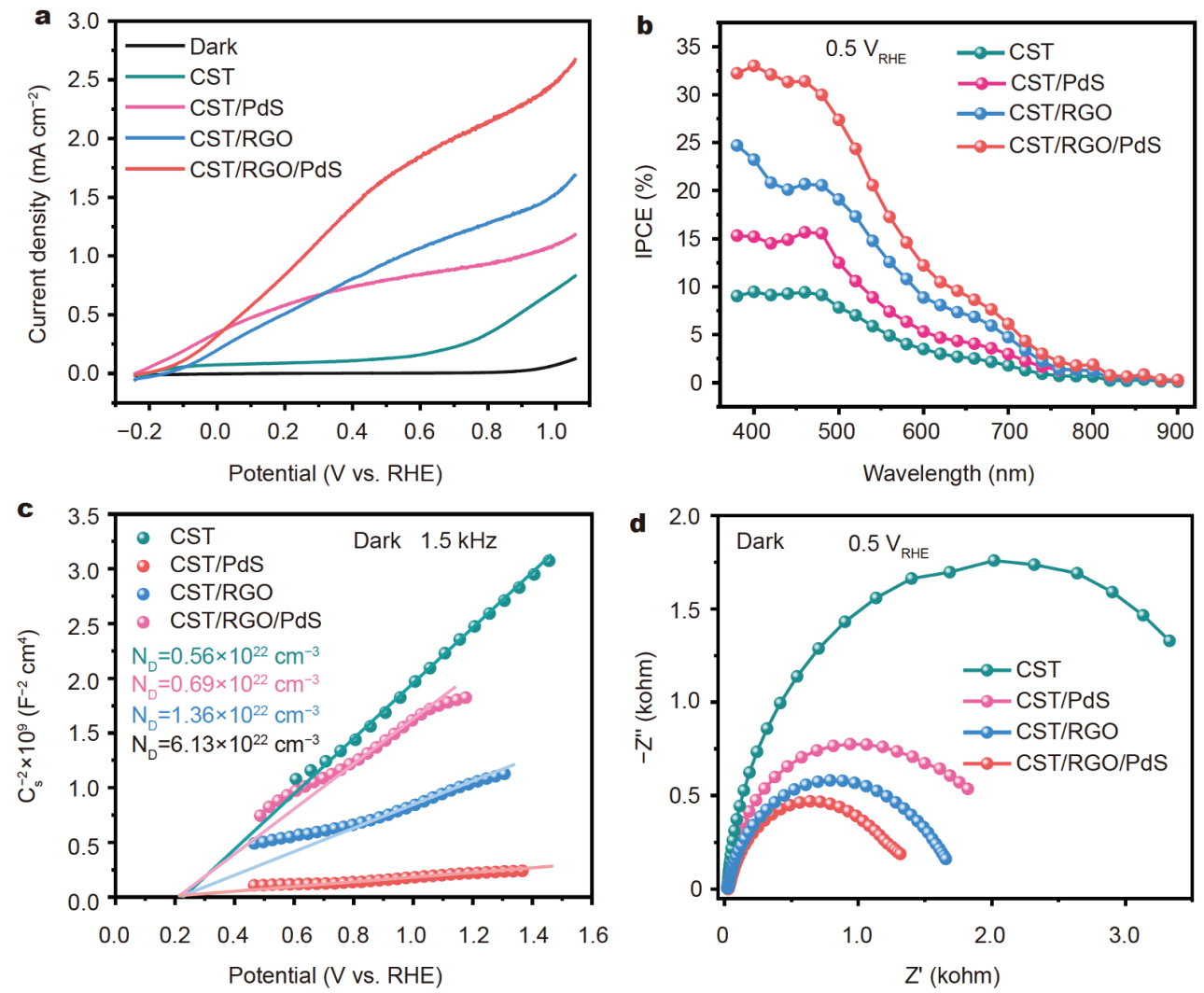

Figure 4 Current-potential curves (a), IPCEs (b), Mott-Schottky plots (c), and Nyquist plots (d) of the CST, CST/PdS, CST/RGO, and CST/RGO/PdS photoanodes. 

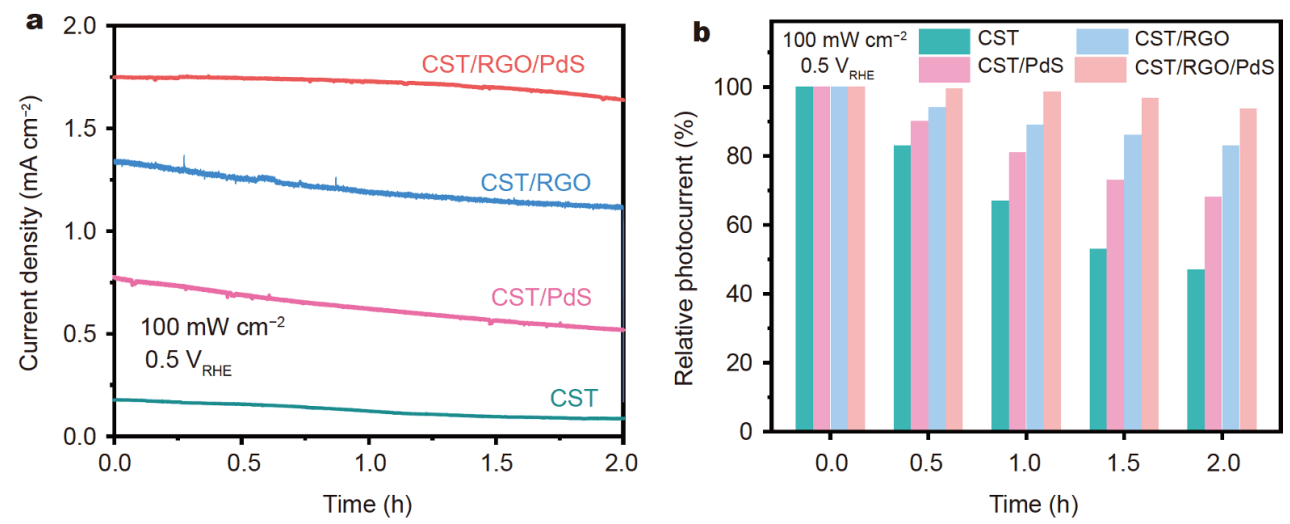

Figure 5 Stability test $(\mathrm{a}, \mathrm{b})$ of the CST, CST/PdS, CST/RGO, and CST/RGO/PdS photoanodes.

photocurrent under the PEC measurement, and only loses about $7 \%$ of its initial photocurrent after $2 \mathrm{~h}$ test. In stark contrast, the CST, CST/RGO, and CST/PdS electrodes suffer from severe decays in PEC performances for each $30 \mathrm{~min}$ of measurement, and lose more than $50 \%$, $30 \%$, and $18 \%$ of their initial photocurrent after $2 \mathrm{~h}$, respectively. Meanwhile, we also conducted a long-term stability test (Fig. S24b, c). Compared with other samples, the RGO/PdS NPs based HEL significantly retards the photocorrosion by means of rapid transfer and consumption of photogenerated holes. For the CST/PdS photoanode, PdS NPs could accelerate the consumption of photogenerated holes, but its improvement in the stability of the catalyst is not significant. The main reason is that photogenerated holes cannot be transferred to the cocatalysts quickly and efficiently due to the lattice-mismatched interface between CST NWs and PdS NPs (Fig. S6). On the contrary, the RGO in RGO/PdS NPs based HEL not only accelerates the extraction of photogenerated holes from CST NWs, but also enables facile interfacial hole transfer toward PdS NPs, thereby effectively improving the PEC stability of CST NWs.

We further characterized the changes in their morphologies and crystal structures at different operation stages. SEM images show that CST and CST/PdS photoanodes undergo tremendous morphological changes after the test, while the morphology of the CST/RGO/PdS photoanode only changes slightly, which are consistent with their decays in current density (Figs S25-S28). In addition, these photoanodes show no difference in XRD patterns after the stability test, except for different extent of oxidation as revealed by XPS spectra (Figs S29-S31). Based on above results, we can confirm that the RGO/PdS NPs based HEL shows superiority in improving the photostability and solar conversion efficiency of photo- anode.

\section{CONCLUSIONS}

In summary, we report the RGO/PdS NPs based HEL as an approach to suppressing the photocorrosion of photoanodes, which are able to rapidly transfer and consume photogenerated holes. The holes generated by CST NWs are transferred by RGO with the high hole-transporting capability to PdS cocatalysts for rapid redox reactions, thus outpacing the competitive photocorrosion process. The photocurrent of the present CST/RGO/PdS photoanode achieves $1.6 \mathrm{~mA} \mathrm{~cm}^{-2}$ at $0.5 \mathrm{~V}_{\mathrm{RHE}}$ under 1-sun irradiation. In addition, this photoanode only loses about $7 \%$ of its initial photocurrent after $2 \mathrm{~h}$ test, which shows excellent stability compared with other photoanodes. This study offers a new route to enhancing the stability of photoanode by introducing an efficient HEL.

Received 4 February 2020; accepted 17 March 2020; published online 21 April 2020

1 Turner JA. A realizable renewable energy future. Science, 1999, 285: 687-689

2 Turner JA. Sustainable hydrogen production. Science, 2004, 305: 972-974

3 Schultz DM, Yoon TP. Solar synthesis: Prospects in visible light photocatalysis. Science, 2014, 343: 1239176

4 Tachibana Y, Vayssieres L, Durrant JR. Artificial photosynthesis for solar water-splitting. Nat Photon, 2012, 6: 511-518

5 McKone JR, Lewis NS, Gray HB. Will solar-driven water-splitting devices see the light of day? Chem Mater, 2014, 26: 407-414

6 Lewis NS. Light work with water. Nature, 2001, 414: 589-590

7 Fujishima A, Honda K. Electrochemical photolysis of water at a semiconductor electrode. Nature, 1972, 238: 37-38

8 Reece SY, Hamel JA, Sung K, et al. Wireless solar water splitting using silicon-based semiconductors and earth-abundant catalysts. Science, 2011, 334: 645-648

9 Chen Y, Zhao S, Wang X, et al. Synergetic integration of $\mathrm{Cu}_{1.94} \mathrm{~S}-$ 
$\mathrm{Zn}_{x} \mathrm{Cd}_{1-x} \mathrm{~S}$ heteronanorods for enhanced visible-light-driven photocatalytic hydrogen production. J Am Chem Soc, 2016, 138: 42864289

10 Li Y, Zhuang TT, Fan F, et al. Pulsed axial epitaxy of colloidal quantum dots in nanowires enables facet-selective passivation. Nat Commun, 2018, 9: 4947

11 Zhuang TT, Liu Y, Li Y, et al. Integration of semiconducting sulfides for full-spectrum solar energy absorption and efficient charge separation. Angew Chem Int Ed, 2016, 55: 6396-6400

12 Zhuang TT, Liu Y, Sun M, et al. A unique ternary semiconductor(semiconductor/metal) nano-architecture for efficient photocatalytic hydrogen evolution. Angew Chem Int Ed, 2015, 54: 11495-11500

13 Liang Q, Guo Y, Zhang N, et al. Improved water-splitting performances of $\mathrm{CuW}_{1-x} \mathrm{Mo}_{x} \mathrm{O}_{4}$ photoanodes synthesized by spray pyrolysis. Sci China Mater, 2018, 61: 1297-1304

14 Kim S, Fisher B, Eisler HJ, et al. Type-II quantum dots: CdTe/ $\mathrm{CdSe}$ (core/shell) and $\mathrm{CdSe} / \mathrm{ZnTe}$ (core/shell) heterostructures. J Am Chem Soc, 2003, 125: 11466-11467

15 Poplawsky JD, Guo W, Paudel N, et al. Structural and compositional dependence of the $\mathrm{CdTe}_{x} \mathrm{Se}_{1-x}$ alloy layer photoactivity in CdTe-based solar cells. Nat Commun, 2016, 7: 12537

16 Rakhshani AE. Electrodeposited CdTe-optical properties. J Appl Phys, 1997, 81: 7988-7993

17 Jasieniak J, Smith L, van Embden J, et al. Re-examination of the size-dependent absorption properties of CdSe quantum dots. J Phys Chem C, 2009, 113: 19468-19474

18 Leatherdale CA, Woo WK, Mikulec FV, et al. On the absorption cross section of CdSe nanocrystal quantum dots. J Phys Chem B, 2002, 106: 7619-7622

19 Pacifici D, Lezec HJ, Atwater HA. All-optical modulation by plasmonic excitation of CdSe quantum dots. Nat Photon, 2007, 1: 402-406

20 Meissner D, Memming R, Kastening B. Photoelectrochemistry of cadmium sulfide. 1. Reanalysis of photocorrosion and flat-band potential. J Phys Chem, 1988, 92: 3476-3483

21 Fermín DJ, Ponomarev EA, Peter LM. A kinetic study of CdS photocorrosion by intensity modulated photocurrent and photoelectrochemical impedance spectroscopy. J Electroanal Chem, 1999, 473: 192-203

22 Allongue P. Primary reactions in the photocorrosion of CdSe through photocapacitance measurements. J Electrochem Soc, 1991, 138: $261-268$

23 Korala L, Wang Z, Liu Y, et al. Uniform thin films of CdSe and $\mathrm{CdSe}(\mathrm{ZnS})$ core(shell) quantum dots by sol-gel assembly: Enabling photoelectrochemical characterization and electronic applications. ACS Nano, 2013, 7: 1215-1223

24 Pan L, Kim JH, Mayer MT, et al. Boosting the performance of $\mathrm{Cu}_{2} \mathrm{O}$ photocathodes for unassisted solar water splitting devices. Nat Catal, 2018, 1: 412-420

25 Wang $\mathrm{H}, \mathrm{Gao} \mathrm{Y}$, Liu J, et al. Efficient plasmonic Au/CdSe nanodumbbell for photoelectrochemical hydrogen generation beyond visible region. Adv Energy Mater, 2019, 9: 1803889

26 Zhang J, Guo Y, Xiong Y, et al. An environmentally friendly Zscheme $\mathrm{WO}_{3} / \mathrm{CDots} / \mathrm{CdS}$ heterostructure with remarkable photocatalytic activity and anti-photocorrosion performance. J Catal, 2017, 356: 1-13

27 Costi R, Young ER, Bulović V, et al. Stabilized CdSe-CoPi composite photoanode for light-assisted water oxidation by transformation of a CdSe/cobalt metal thin film. ACS Appl Mater
Interfaces, 2013, 5: 2364-2367

28 Yan $\mathrm{H}$, Yang J, Ma G, et al. Visible-light-driven hydrogen production with extremely high quantum efficiency on Pt-PdS/CdS photocatalyst. J Catal, 2009, 266: 165-168

29 Ye $\mathrm{KH}$, Wang $\mathrm{Z}$, Li $\mathrm{H}$, et al. A novel $\mathrm{CoOOH} /(\mathrm{Ti}, \mathrm{C})-\mathrm{Fe}_{2} \mathrm{O}_{3}$ nanorod photoanode for photoelectrochemical water splitting. Sci China Mater, 2018, 61: 887-894

30 Peng Y, Ji J, Chen D. Ultrasound assisted synthesis of $\mathrm{ZnO} /$ reduced graphene oxide composites with enhanced photocatalytic activity and anti-photocorrosion. Appl Surf Sci, 2015, 356: 762-768

31 Li Q, Li X, Wageh S, et al. CdS/graphene nanocomposite photocatalysts. Adv Energy Mater, 2015, 5: 1500010

32 Xie G, Zhang K, Guo B, et al. Graphene-based materials for hydrogen generation from light-driven water splitting. Adv Mater, 2013, 25: 3820-3839

33 Yang Y, Wang K, Liang HW, et al. A new generation of alloyed/ multimetal chalcogenide nanowires by chemical transformation. Sci Adv, 2015, 1: e1500714

34 Qian HS, Yu SH, Gong JY, et al. High-quality luminescent tellurium nanowires of several nanometers in diameter and high aspect ratio synthesized by a poly (vinyl pyrrolidone)-assisted hydrothermal process. Langmuir, 2006, 22: 3830-3835

35 Tsuji I, Kato $\mathrm{H}$, Kobayashi $\mathrm{H}$, et al. Photocatalytic $\mathrm{H}_{2}$ evolution reaction from aqueous solutions over band structure-controlled $(\operatorname{AgIn})_{x} \mathrm{Zn}_{2(1-x)} \mathrm{S}_{2}$ solid solution photocatalysts with visible-light response and their surface nanostructures. J Am Chem Soc, 2004, 126: 13406-13413

36 Maiolo JR, Atwater HA, Lewis NS. Macroporous silicon as a model for silicon wire array solar cells. J Phys Chem C, 2008, 112: 61946201

37 Liu M, Lyons JL, Yan D, et al. Semiconductor-based photoelectrochemical water splitting at the limit of very wide depletion region. Adv Funct Mater, 2015, 26: 219-225

38 Hill JC, Landers AT, Switzer JA. An electrodeposited inhomogeneous metal-insulator-semiconductor junction for efficient photoelectrochemical water oxidation. Nat Mater, 2015, 14: 1150-1155

39 Zhang Z, Dua R, Zhang L, et al. Carbon-layer-protected cuprous oxide nanowire arrays for efficient water reduction. ACS Nano, 2013, 7: 1709-1717

40 Liu Q, He J, Yao T, et al. Aligned $\mathrm{Fe}_{2} \mathrm{TiO}_{5}$-containing nanotube arrays with low onset potential for visible-light water oxidation. Nat Commun, 2014, 5: 5122

Acknowledgements This work was supported by the National Natural Science Foundation of China (51732011, 21431006, 21761132008, 81788101 and 11227901), the Foundation for the Innovative Research Groups of the National Natural Science Foundation of China (21521001), the Key Research Program of Frontier Sciences, CAS (QYZDJ-SSW-SLH036), the National Basic Research Program of China (2014CB931800), the Users with Excellence and Scientific Research Grant of Hefei Science Center of CAS (2015HSC-UE007). This work was partially carried out at the USTC Center for Micro and Nanoscale Research and Fabrication.

Author contributions Yu SH supervised the project and wrote the paper. Liu GQ carried out the experiments, analyzed the results, and wrote the paper. Xu Q, Hu J, and Zhu JF performed the XPS and UPS measurements. Li Y and Fan FJ provided the TA measurement and helpful suggestion about the paper. Yang Y analyzed the crystal structure 
and growth mechanism. Peng JL performed the SEM measurements. Li $\mathrm{Y}$ assisted with the photocatalysis experiments. All authors contributed general discussion.

Conflict of interest The authors declare no conflict of interest. Supplementary information Supporting data are available in the online version of the paper.

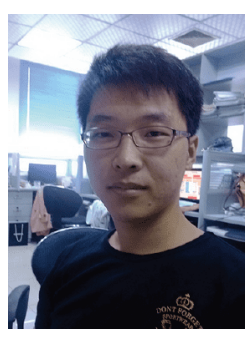

Guo-Qiang Liu received his bachelor degree majored in chemistry from Shandong University, China in 2014. Then he joined the University of Science and Technology of China (USTC) and conducted research under the supervision of Prof. Shu-Hong Yu. His research interest is the synthesis of 1D metal chalcogenide nanowires as efficient photoelectrochemical catalysts.

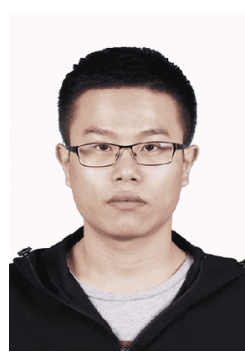

Yi Li received his $\mathrm{PhD}$ degree from USTC in 2019. Currently, he is a post-doctoral fellow in Prof. Shu-Hong Yu's group at USTC. His research interests include the design and synthesis of novel 1D semiconductor nanomaterials and their heterostructures for solar-to-fuel conversion.

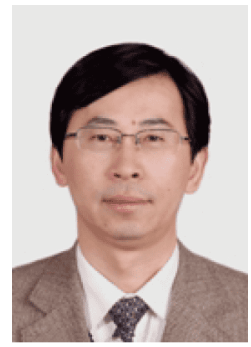

Shu-Hong Yu completed his PhD from USTC Currently, he leads the Division of Nanomaterials \& Chemistry at Hefei National Laboratory for Physical Sciences at Microscale, USTC. He was elected as an academician of the Chinese Academy of Sciences in 2019. His current research interests include bio-inspired synthesis, self-assembly of new nanostructured materials and nanocomposites, and their related properties.

\section{基于还原氧化石墨烯/硫化钯的空穴提取层助力 光阳极的抗光腐蚀研究}

刘国强 ${ }^{1 \dagger}$, 李效 ${ }^{1 \dagger}$, 阳缘 ${ }^{1}$, 㚞逢佳 ${ }^{2}$, 丁光浩 ${ }^{1}$, 伍亮 ${ }^{1}$, 胡俊 ${ }^{3}$, 彭金兰 ${ }^{4}$, 徐倩 $^{3},{\text { 朱俊发 }{ }^{3}, \text { 俞书宏 }}^{1^{*}}$

摘要 光电化学(PEC)产氢作为产生清洁、可持续能源的理想途 径, 引起了科学家们极大的兴趣. 然而, 光阳极的不稳定性及低能量 转换效率使其实际应用受到阻碍. 本文通过设计空穴提取层(HEL) 加速光生空穴的转移与消耗, 进而改善光阳极的不稳定性. HEL 由 还原氧化石墨烯(RGO)和其他助催化剂构成, 可分别用于快速转移 空穴和加速空穴消耗. 本文展示了基于CdSeTe纳米线和基于RGO/ PdS纳米颗粒的HEL组成的高稳定性光阳极. 该光阳极具有出色的 耐光腐蚀性能, 可在 $0.5 \mathrm{~V}_{\mathrm{RHE}}$ 下稳定产氢 $2 \mathrm{~h}$ 以上. 